\title{
OPEN Hyperglycemia-induced VEGF and ROS production in retinal cells is inhibited by the mTOR inhibitor, rapamycin
}

\author{
Teruyo Kida ${ }^{\varpi}$, Hidehiro Oku, Sho Osuka, Taeko Horie \& Tsunehiko Ikeda
}

Determine the impact of the mTOR inhibitor, rapamycin, on the hyperglycemia-induced expression of vascular endothelial growth factor (VEGF) and the production of reactive oxygen species (ROS) in retinal cells. Rats made hyperglycemic for 8 weeks by streptozotocin, as well as control rats, received i.p. rapamycin ( $1 \mathrm{mg} / \mathrm{kg}$ ) for 3 days prior to immunostaining of their retinas with anti-VEGF and anti-glial fibrillary acidic protein (GFAP) and measuring retinal protein levels of VEGF and GFAP by Western blotting. In other experiments, flow cytometry analysis of ethidium fluorescence determined intracellular ROS levels in the absence or presence of rapamycin $(1 \mu \mathrm{M})$ under normoglycemic $(5.5 \mathrm{mM})$ and hyperglycemic ( $25 \mathrm{mM}$ ) conditions in a rat retinal Müller cell line (TR-MUL5) and primary human retinal microvascular endothelial cells (HRMECs). In the diabetic retina, VEGF was elevated and colocalized with the glial marker, GFAP, whose level was also elevated. Treatment with rapamycin inhibited the diabetes-induced VEGF and GFAP increases. We also found that raising extracellular glucose from $5.5 \mathrm{mM}$ to $25 \mathrm{mM}$ resulted in significant rapamycin-sensitive increases in the ROS levels of TR-MUL 5 cells and HRMECs. In rat retina, rapamycin attenuates the diabetes-induced VEGF overexpression, and in cultured Müller cells and HRMECs, inhibits the hyperglycemia-induced boost ROS.

Hyperglycemia is the primary cause of vascular complications occurring in individuals with diabetes ${ }^{1-3}$. An important microvascular complication is sight-threatening diabetic retinopathy ${ }^{4-6}$ in which chronic hyperglycemia is associated with increased expression of vascular endothelial growth factor (VEGF), which is known to be synthesized in the retinal Müller glial cells, and elevated levels of reactive oxygen species (ROS), which are produced by retinal cells including the glia and vascular endothelium ${ }^{7-12}$.

These responses of the diabetic retina are likely to be interrelated since exposure to VEGF is known to boost the production of ROS by the Müller cells and also the cells of the vascular endothelium ${ }^{13,14}$. Even though the physiological roles of VEGF in the retina remain uncertain, its upregulation is well-established to also cause a breakdown of the blood-retinal barrier, whose integrity is essential for optimal retinal function. Furthermore, although redox signaling is plays a key role in the physiological regulation of blood flow ${ }^{9,15,16}$, ROS overproduction is thought to contribute to the endothelial dysfunction and neurodegeneration observed in diabetic retinopathy ${ }^{3,17-19}$.

In this study, we considered the working hypothesis that the mTOR signaling pathway plays a role in the pathological upregulation of retinal VEGF and ROS observed in the course of diabetes. Not only has elevated mTOR activity been observed in diabetes ${ }^{20}$, but the mTOR pathway is involved in the upregulation of VEGF. To begin assess our working hypothesis, we first performed immunohistochemistry to compare the expression of VEGF and the glial marker, GFAP, in the diabetic retina and the effects of rapamycin, an immunosuppressive and anti-cancer drug that is reported to suppress the action of $\mathrm{mTOR}^{21,22}$. We also used immunoblots to determine whether VEGF and GFAP expression were increased in the retina of a streptozotocin (STZ)-induced rat model of diabetes. In addition, we conducted a flow cytometry study to determine the rapamycin effects on ROS levels in cultured TR-MUL5 cells, a rat Müller cell line, and primary human retinal microvascular endothelial cells (HRMECs) as well, following exposure to physiological (5.5 $\mathrm{mM})$ and high glucose $(25 \mathrm{mM})$ conditions.

In this study we show that rapamycin effectively attenuates the hyperglycemia-induced overexpression of VEGF and GFAP in the rat retina. In addition, this mTOR inhibitor suppressed the high glucose-induced 
increases in ROS in cultured Müller cells as well as HRMECs whose dysfunction and degeneration is a wellestablished feature of diabetic retinopathy.

\section{Materials and methods}

Animals. Twenty-four 9-week-old male Wistar rats weighing between 180 and 200 g were purchased from Japan SLC Inc. (Shizuoka, Japan) and housed in an air-conditioned room at approximately $23{ }^{\circ} \mathrm{C}$ with $60 \%$ humidity. They were maintained on a 12-h light/dark cycle and were provided with food and water ad libitum. They were handled in accordance with the ARVO Statement for the Use of Animals in Ophthalmic and Vision Research. Our experimental protocols conformed to the Animal Research: Reporting In Vivo Experiments (ARRIVE) guidelines ${ }^{23}$ and was approved by the Osaka Medical College Committee on the Use and Care of Animals (Approval number: 2019-100).

Chemicals. All chemicals were obtained from Sigma-Aldrich (St. Louis, MO, USA) unless otherwise specified. Rapamycin was purchased from Tokyo Chemical Industry (Tokyo, Japan). A rapamycin dose of $1 \mathrm{mg} / \mathrm{kg}$ has been confirmed as neuroprotective and renoprotective $e^{24,25}$, so we chose this concentration for our experiments. In addition, a rapamycin concentration of $1 \mu \mathrm{M}$, which was converted from the dose of the rapamycin confirmed in vivo study, was used for in vitro study.

Anesthesia and euthanasia. All procedures were performed anesthesia using intraperitoneal injection of medetomidine $(0.75 \mathrm{mg} / \mathrm{kg})$, midazolam $(4 \mathrm{mg} / \mathrm{kg})$, and butorphanol $(5 \mathrm{mg} / \mathrm{kg})$ as described previously ${ }^{26}$. Rats were euthanized by exposure to $\mathrm{CO}_{2}$ with wood shaving bedding.

Induction of diabetes. Diabetic and non-diabetic rats were produced as described previously ${ }^{27}$. Diabetes was induced by administering a single $60 \mathrm{mg} / \mathrm{kg}$ intraperitoneal injection of streptozotocin in $10 \mathrm{mM}$ sodium citrate buffer, $\mathrm{pH} 4.5$, to each rat after an overnight fast. As controls, nondiabetic animals received an injection of citrate buffer only. Animals with blood glucose levels higher than $250 \mathrm{mg} / \mathrm{dL}$ at $24 \mathrm{~h}$ after injections were considered diabetic. All experiments were conducted 8 weeks after the induction of diabetes. The blood glucose was checked again at the time of the experiments to ensure that the rats showed hyperglycemia. The blood glucose level at the time of sacrifice was $384.8 \pm 23.9 \mathrm{mg} / \mathrm{dL}$.

Intraperitoneal injection of rapamycin. Rapamycin ( $1 \mathrm{mg} / \mathrm{kg}$ ) (Tokyo Chemical Industry, Tokyo, Japan) was injected intraperitoneally once a day for 3 days in STZ-induced diabetic and normoglycemic rats. Animals received general anesthesia as previously described, and perfused fixation was performed $24 \mathrm{~h}$ after the intraperitoneal injections.

Immunohistochemistry of retinal slices. All procedures were performed as described previously ${ }^{27}$. Twelve rats were deeply anesthetized, and saline was perfused through the heart, followed by $4 \%$ paraformaldehyde (PFA) in $0.1 \mathrm{M}$ phosphate-buffered saline (PBS, pH 7.4). The retinas were carefully removed and post-fixed in $4 \%$ PFA in PBS overnight. After washing with PBS, the retinal tissues were immersed in $30 \%$ sucrose for 2-3 days at $4{ }^{\circ} \mathrm{C}$, embedded in OCT compound (Sakura Finetechnical, Tokyo, Japan), and cut into $10 \mu \mathrm{m}$-thick frozen sections with a cryostat. After blocking with $1 \%$ normal goat serum plus $1 \%$ BSA and $0.1 \%$ triton X-100 in PBS, the retinal sections were incubated overnight at $4{ }^{\circ} \mathrm{C}$ with the following primary antibodies: mouse monoclonal anti-VEGF (1:500, sc-7269, Santa Cruz Biotechnology, Inc., Dallas, TX, U.S.A.) and rabbit polyclonal anti-GFAP (1:500, AB5804, Merck Millipore, Billerica, MA, U.S.A.). These sections were incubated for $2 \mathrm{~h}$ at $37^{\circ} \mathrm{C}$ with the appropriate Alexa 594- (A11012) or Alexa 488- (A11001) conjugated secondary antibodies (1:500; Invitrogen, Carlsbad, CA, U.S.A.). The processed sections were photographed with a fluorescent microscope (BZ-X700, Keyence, Osaka, Japan) by using Z-scan of 35 images at $0.5 \mu \mathrm{m}$ intervals in each retinal section under the same exposure time.

Protein levels of VEGF and GFAP in retinas by western blotting. All procedures were performed as described previously ${ }^{27,28}$. Retinas were excised from the eyes and homogenized in lysis buffer containing $1 \mathrm{mM}$ phenyl methanesulfonyl fluoride, $10 \mu \mathrm{M}$ pepstatin $\mathrm{A}, 10 \mu \mathrm{M}$ leupeptin, $10 \mu \mathrm{M}$ aprotinin, $0.1 \%$ sodium dodecyl sulfate (SDS), $1 \%$ Nonidet P-40, 5\% sodium deoxy cholate, $50 \mathrm{mM}$ Tris- $\mathrm{HCl}$ ( $\mathrm{pH} 7.6$ ), and $150 \mathrm{mM}$ sodium chloride. The suspension was centrifuged, and the total protein concentration in the supernatant was determined using the Lowry method (DC Protein Assay Reagent, Bio-Rad, Hercules, CA, USA). Samples were separated on a 10-12\% SDS-polyacrylamide gel and blotted onto PVDF membranes. The membranes were then blocked with 5\% skim milk in Tris-buffered saline, $\mathrm{pH} 7.4$, with $0.1 \%$ Tween 20 (TBS-T) followed by an overnight incubation at $4{ }^{\circ} \mathrm{C}$ with a rabbit polyclonal anti-VEGF $(1: 1000$, sc-507, Santa Cruz Biotechnology) or GFAP (1:1000, 04-1031, Merck Millipore). Tubulin ( $\alpha$-tubulin, 1:1000; Merck Millipore) was used as an internal control. The membranes were washed in TBS-T followed by incubation with a peroxidase-conjugated goat anti-rabbit (W401B) or mouse IgG (W402B, 1:2500, Promega, Madison, WI, USA) secondary antibody for $2 \mathrm{~h}$ at $37^{\circ} \mathrm{C}$. The protein bands were visualized following the addition of an ECL Plus Western blotting detection system (GE Healthcare Life Sciences, Little Chalfont, UK). Protein band densities were measured with a luminescent image analyzer (LAS-3000, Fujifilm, Tokyo, Japan). Relative protein levels were quantified using the embedded software (Multi Gauge version 3.0) and standardized according to $\alpha$-tubulin protein levels. 
Cell cultures. A rat retinal Müller cell line (TR-MUL5) was obtained from Fact, Inc., Sendai, Japan ${ }^{29,30}$, and primary human retinal microvascular endothelial cells (HRMECs) were purchased from Cell Systems Corporation, Kirkland, WA, USA. The TR-MUL5 cells and the HRMECs were cultured in Dulbecco's Modified Eagle's Medium (DMEM) containing a physiological concentration of $5.5 \mathrm{mM}$ glucose supplemented with $10 \%$ fetal bovine serum (FBS) at $37^{\circ} \mathrm{C}$ in a humidified atmosphere of $5 \% \mathrm{CO}_{2} /$ air. TR-MUL5 cells at passages 18 and 21 , and HRMECs at passages 4 and 6 were used in this study. These cells were maintained in DMEM that contained $5.5 \mathrm{mM}$ glucose. Before reaching confluence, the media was changed to the control media (5.5 mM glucose in DMEM) or the high glucose media (25 mM glucose in DMEM, D6429) to compare ROS levels, as described later. Detailed procedures and treatment protocols for each experiment are described in subsequent sections.

Immunostaining of cultured Müller cells. To determine the effect of the mTOR inhibitor rapamycin on the expression of VEGF and GFAP in the TR-MUL5 cells under high glucose condition, the cells that were incubated in high glucose $(25 \mathrm{mM})$ media with and without rapamycin were examined by immunocytochemistry. After fixation by $4 \%$ formaldehyde, the cells were incubated with primary antibodies of mouse monoclonal anti-VEGF (sc-7269, 1:100, Santa Cruz Biotechnology, Inc., Dallas, TX, U.S.A.) and rabbit polyclonal anti-GFAP (AB5804, 1:100, Merck Millipore, Billerica, MA, U.S.A.) overnight at $4{ }^{\circ} \mathrm{C}$. After rinsing by PBS and blocking, these cells were incubated for $2 \mathrm{~h}$ at room temperature in Alexa 594 (A11012) or Alexa 488- (A11001) conjugated to the appropriate secondary antibodies (Invitrogen, Carlsbad, CA, U.S.A.) diluted by 1:500. Nuclei were stained with 4',6-diamidino-2-phenylindole (DAPI, 1:1000, Dojindo, Inc. Kumamoto, Japan). The processed samples were photographed with a fluorescence microscope (BZ-X700, Keyence, Osaka, Japan).

Flow cytometry analyses of ROS formation in müller cells and HRMECs. We examined the generation of ROS in cultured Müller cells (TR-MUL5) and HRMECs by treating them with hydroethidine and measuring ethidium fluorescence by flow cytometry (EC800 cell analyzer, SONY, Tokyo, Japan). After a few passages of TR-MUL5 and HRMECs, the media was changed to the control media (5.5 mM glucose in DMEM) or the high glucose media (25 mM glucose in DMEM, D6429) with $10 \%$ fetal bovine serum (FBS). The cells were incubated overnight at $37^{\circ} \mathrm{C}$ with or without rapamycin $(1 \mu \mathrm{M})$ under physiological $(5.5 \mathrm{mM})$ and high-glucose $(25 \mathrm{mM})$ conditions for $48 \mathrm{~h}$. The cells were cultured in a medium lacking 10\% FBS and were serum-deprived overnight before the following FACS assay. Cells were harvested via trypsinization, centrifuged at $1000 \times g$ for $5 \mathrm{~min}$, re-suspended in phenol red-free DMEM, and incubated with hydroethidine, a fluorogenic probe, for $20 \mathrm{~min}$ at $37^{\circ} \mathrm{C}$. Cell densities were adjusted to $2.0 \times 10^{5}$ cells $/ \mathrm{mL}$. Hydroethidine is oxidized by ROS to form ethidium, a fluorescent product, which is then retained intracellularly to allow a semi-quantitative estimation of intracellular ROS levels ${ }^{31,32}$. The intracellular levels of ROS were measured using an EC800 cell analyzer at $488 \mathrm{~nm}$ excitation and 590-610 $\mathrm{nm}$ emission wavelengths. The acquisition and analysis software programs on the EC800 was used to acquire and quantify the fluorescence intensities. This assay was performed to determine the levels of ROS generated by the cultured cells in control and high glucose media in the presence or absence of rapamycin $(1 \mu \mathrm{M})$. In addition, the cultured Müller cells (TR-MUL5) were also incubated overnight with or without VEGF $(5 \mathrm{ng} / \mathrm{mL})$ and rapamycin under physiological $(5.5 \mathrm{mM})$ and high-glucose $(25 \mathrm{mM})$ conditions to determine ROS production by VEGF and the effects of rapamycin on this ROS production. The ethidium fluorescence in TR-MUL5 cells exposed to VEGF and/or rapamycin under physiological ( $5.5 \mathrm{mM})$ and high-glucose $(25 \mathrm{mM})$ conditions was also measured by flow cytometry.

Statistical analyses. The means and standard deviation of the means were calculated. Unless otherwise noted, two-tailed Student's $t$-tests were used. The level of significance was set at $P<0.05$. Multiple groups in Figs. 4 and 5 were compared ANOVA analysis; Bonferroni correction.

Ethical approval. All applicable guidelines of ARVO Statement for the care and use of animals were followed. Our experimental protocols conformed to the Animal Research: Reporting In Vivo Experiments (ARRIVE) guidelines ${ }^{23}$.

All procedures performed in studies involving animals were in accordance with the ethical standards of the Osaka Medical College Committee on the Use and Care of Animals (Approval number: 2019-100) at which the studies were conducted.

\section{Results}

Immunohistochemistry of retinal slices. Compared with the nondiabetic control rats, immunoreactivity to VEGF was increased in the STZ-induced diabetic rats. As shown in Fig. 1, VEGF immunoreactivity was seemed to be higher in the diabetic rat retinas than in the controls. Injection of rapamycin into the nondiabetic rats seemed to decrease VEGF immunoreactivity. In the diabetic retina, VEGF expression seemed to be intensified mainly in these internal retinal layers. Rapamycin likely decreased the intensity in diabetic rats.

Immunoreactivity to GFAP seemed to be higher in the diabetic rat retina than in the control retina. The observed longitudinal pattern of immunoreactivity to GFAP in the inner plexiform layer (IPL), the inner nuclear layer (INL), the outer plexiform layer (OPL), and the outer nuclear layer (ONL) suggested that these GFAP expression may involve Müller cells. Double staining with VEGF and GFAP demonstrated that the expression of VEGF was co-localized with GFAP expression especially in the diabetic rat retinas. Rapamycin seemed to inhibit the increases in GFAP expression in the diabetic retina. 


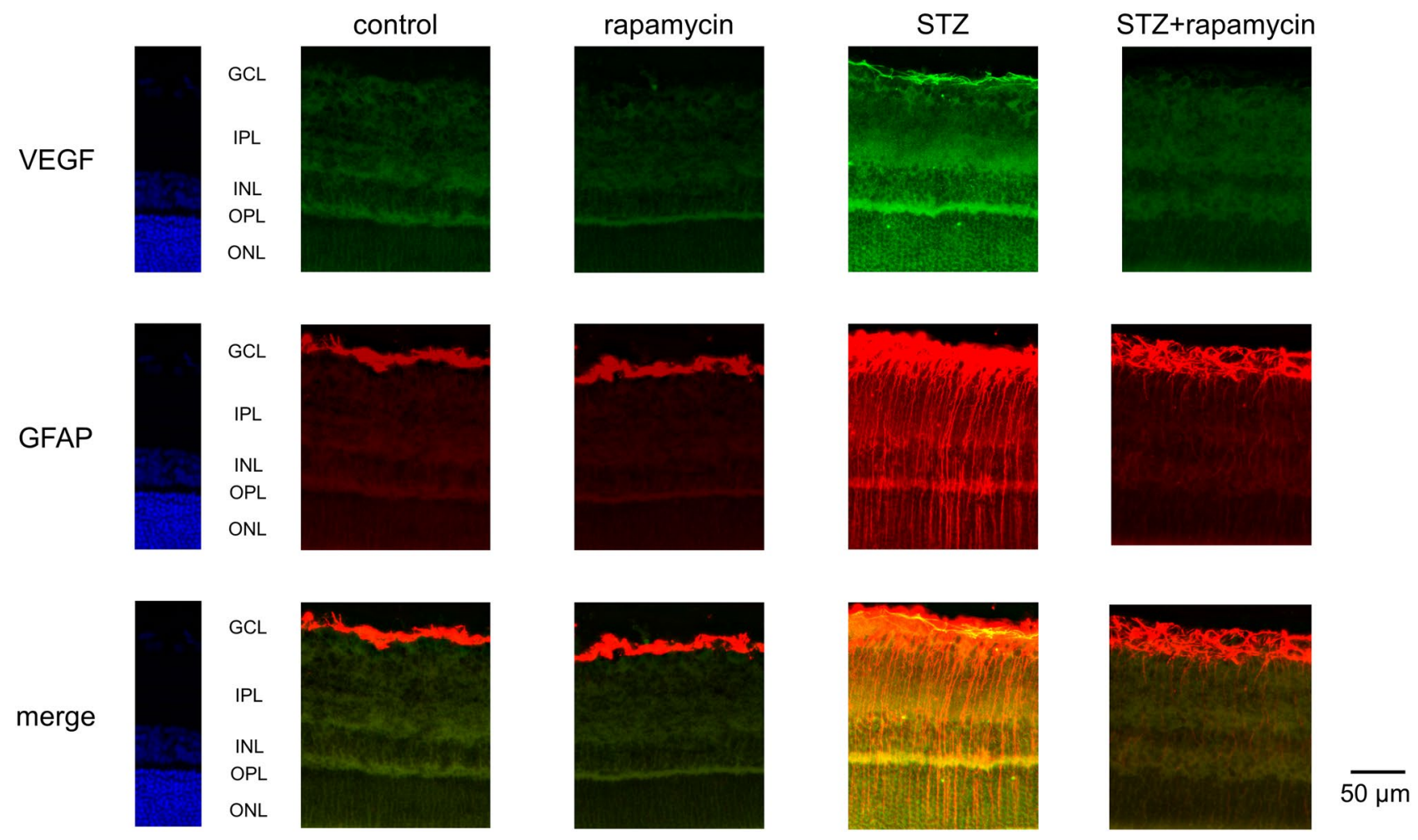

Figure 1. Representative photomicrographs of retinal tissues stained immunohistochemically with anti-VEGF (green) and anti-GFAP (red) antibodies. VEGF immunoreactivity was higher in the diabetic retina sections than in the control retinas, and expression of VEGF was colocalized with GFAP.

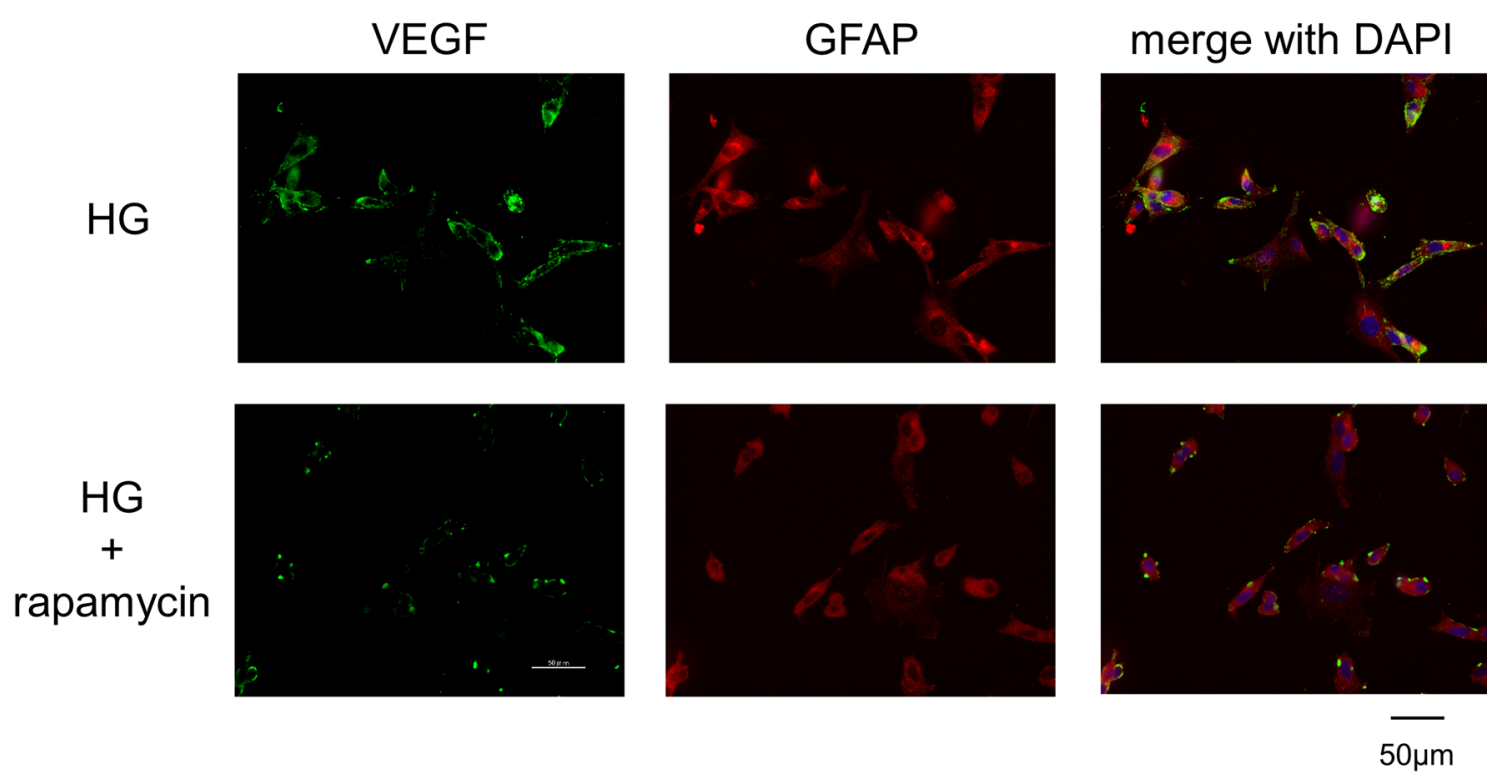

Figure 2. Immunostaining of VEGF and GFAP in TR-MUL5 cells. Expression of VEGF and GFAP in cells cultured in high glucose medium (HG) seemed to be reduced by the mTOR inhibitor rapamycin (HG + rapamycin).

Immunostaining of cultured Müller cells. Representative photomicrographs of immunostaining with anti-VEGF (green) and anti-GFAP (red) in the TR-MUL5 cells are shown in Fig. 2. Immunoreactivities to VEGF and GFAP in high glucose condition seemed to be depressed by the mTOR inhibitor rapamycin. 


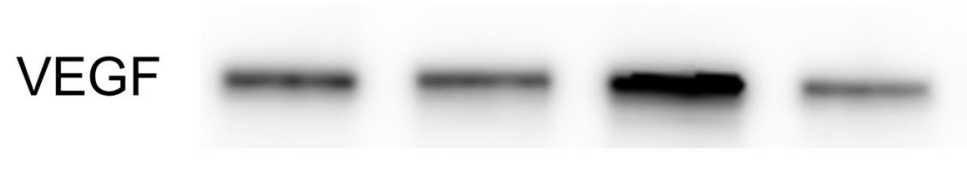

\section{tubulin}
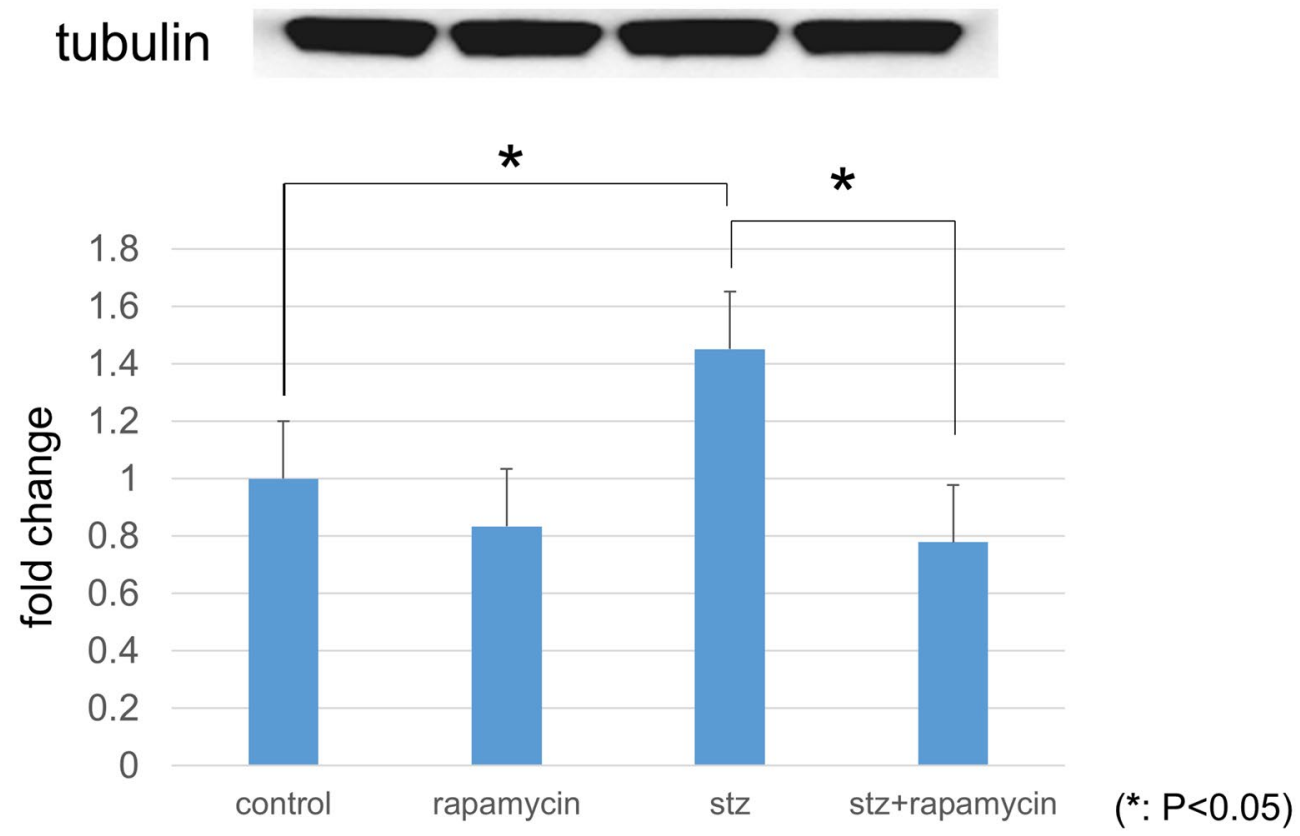

Figure 3. Protein levels of VEGF in retinas determined with Western blotting. VEGF expression was significantly higher in retinas obtained from STZ-induced diabetic rats than from nondiabetic controls. The increased VEGF expression was suppressed by treatment with the mTOR inhibitor rapamycin $(P<0.05, \mathrm{n}=3$ each).

Protein levels of VEGF and GFAP in retinas by western blotting. The protein levels of VEGF in the STZ-induced diabetic retina were significantly up-regulated to $145.2 \%$ compared to the control retina $(P<0.0001)$. These increases were significantly suppressed by rapamycin $(P<0.0001)$ (Fig. $3, \mathrm{n}=3$ each).

Protein levels of GFAP in the diabetic retina were significantly higher $(28.1 \%)$ than in the control retina $(P=0.0004)$. The increases were significantly suppressed by rapamycin $(P<0.0001)$ (Fig. $4, \mathrm{n}=3$ each).

Flow cytometry analyses of intracellular production of ROS in TR-MUL5 and HRMECs. Figure 5 shows the effects of rapamycin $(1 \mu \mathrm{M})$ treatment on hydroethidine fluorescence in cultured rat Müller cells (TR-MUL5) under physiological $(5.5 \mathrm{mM})$ and high-glucose $(25 \mathrm{mM})$ conditions. Flow cytometry analyses showed significant increase in intracellular levels of ROS in TR-MUL5 under the high- glucose condition, and these increases were significantly suppressed by rapamycin. This figure also shows the effects of rapamycin on VEGF in ROS production of TR-MUL5 under physiological $(5.5 \mathrm{mM})$ and high-glucose $(25 \mathrm{mM})$ conditions. We performed this assay to determine the effect of rapamycin on the ROS production of cultured Müller cells (TR-MUL5) exposed to VEGF. Flow cytometry analyses showed significant increases in the intracellular levels of ROS in TR-MUL5 cells exposed to VEGF, and these increases were significantly suppressed by rapamycin $(P<0.0018$, the Bonferroni correction).

Figure 6 shows the intracellular ROS levels in HRMECs exposed to rapamycin under physiological (5.5 mM) and high-glucose $(25 \mathrm{mM})$ conditions. Significant increases in the ROS levels in HRMECs under high-glucose condition were observed, and these increases were significantly suppressed by rapamycin $(P<0.0083$, the Bonferroni correction).

\section{Discussion}

A key finding of this study is that the diabetes-induced increase in VEGF expression in the diabetic rat retina is inhibited by the mTOR inhibitor, rapamycin. This enzymatic inhibitor also blocked the up-regulation of GFAP by glial cells of the diabetic retina. An additional novel observation is that rapamycin inhibits the hyperglycemiainduced increase in ROS in cultured Müller cells as well as HERMCs. We also found that under hyperglycemic conditions, the VEGF-induced increase in Müller cell ROS was also suppressed by rapamycin.

The increase in VEGF levels in the diabetic retina have been previously reported ${ }^{19,27,33-35}$, and this response was suppressed by rapamycin. Generally, mTOR coordinates protein synthesis, mitochondrial energy production ${ }^{36}$. 
GFAP

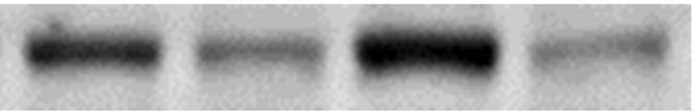

tubulin

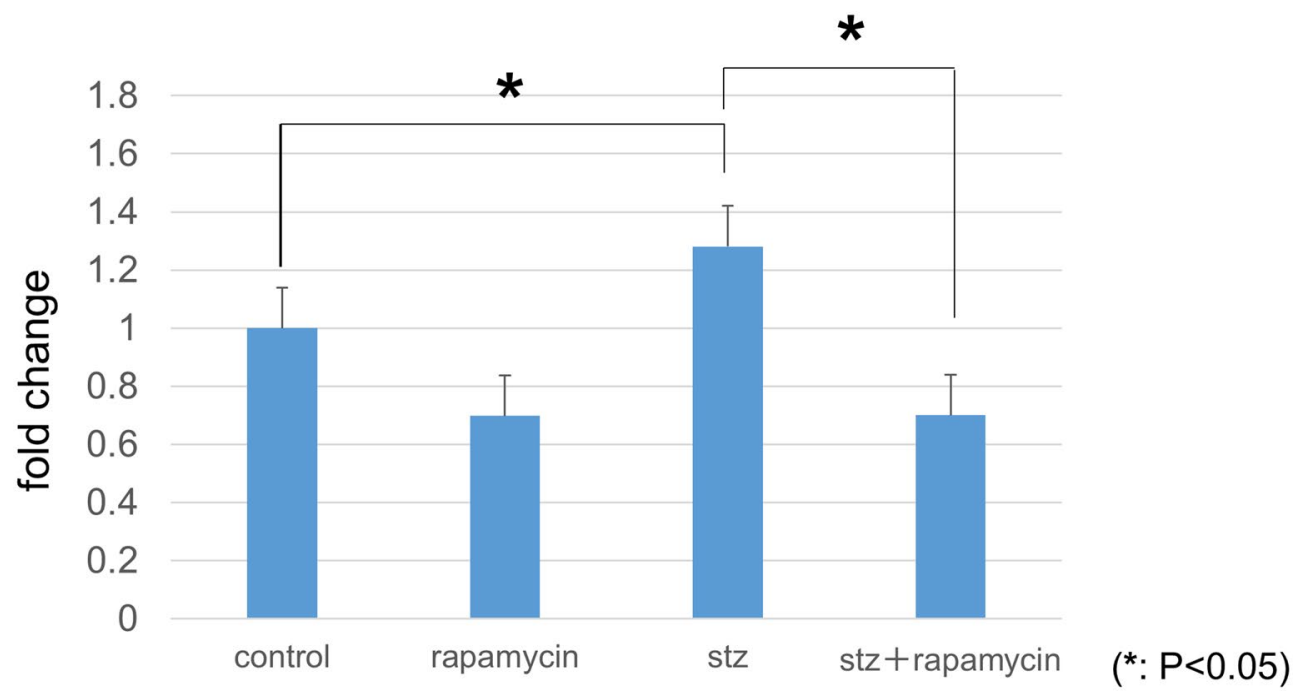

Figure 4. Protein levels of GFAP in retinas determined with Western blotting. GFAP expression was significantly higher in retinas obtained from STZ-induced diabetic rats than from nondiabetic controls. The increased GFAP expression was suppressed by rapamycin $(P<0.05, \mathrm{n}=3$ each).

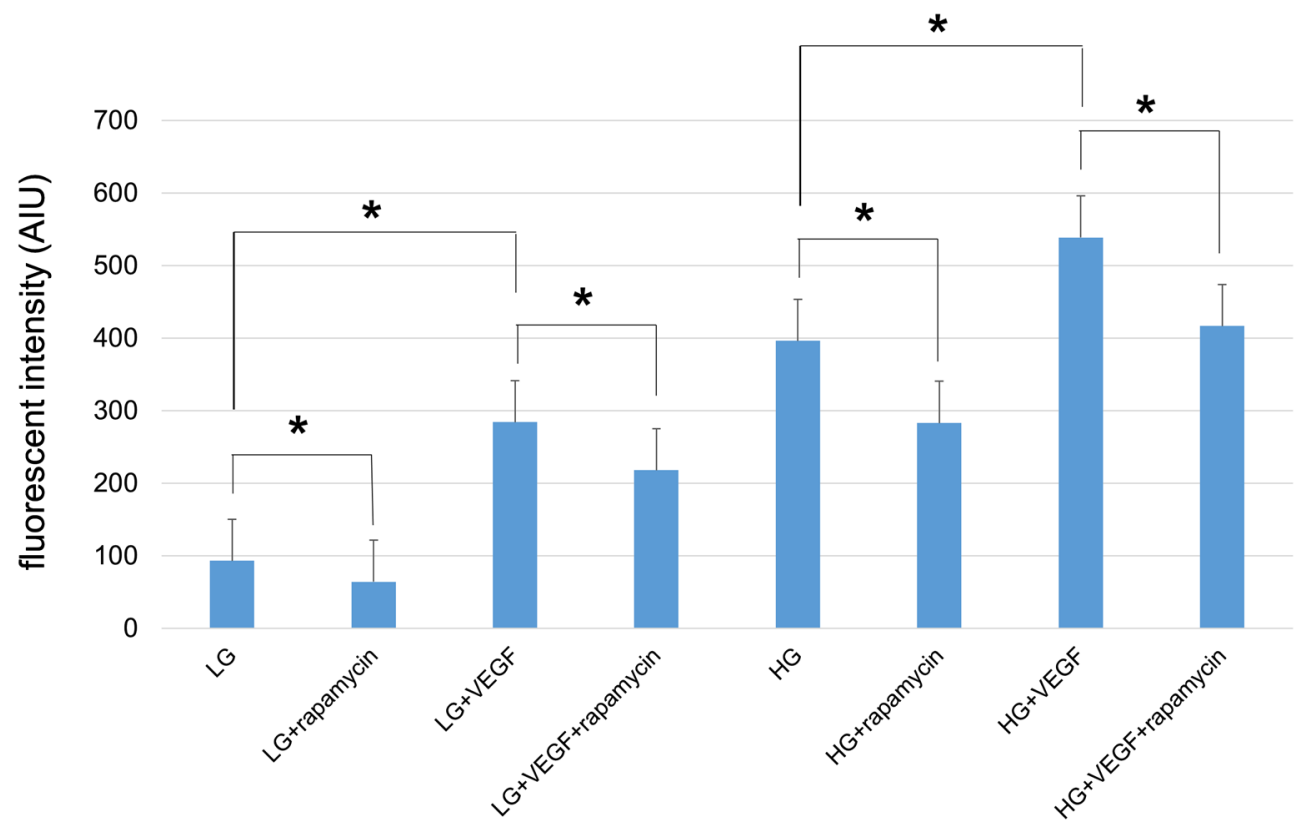

Figure 5. ROS levels in cultured Müller cells (TR-MUL5) exposed to rapamycin and/or VEGF under physiological $(5.5 \mathrm{mM})$ and high-glucose $(25 \mathrm{mM})$ conditions. Flow cytometry analyses showed significant increases in intracellular ROS levels in TR-MUL5 under a high-glucose condition, and these increases were significantly suppressed by rapamycin. In addition, flow cytometry analyses showed significant increases in intracellular levels of ROS in TR-MUL5 exposed to VEGF, and these increases were significantly suppressed by rapamycin $(P<0.0018$, the Bonferroni correction). 


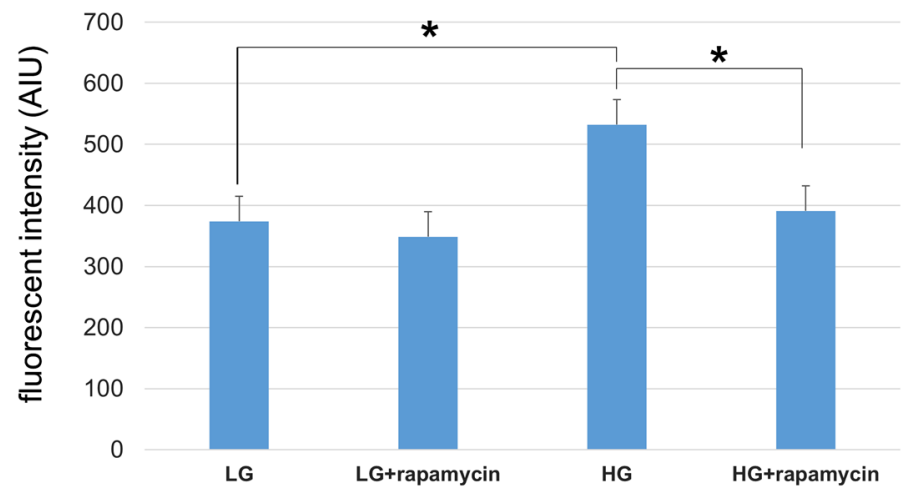

Figure 6. ROS levels in primary human retinal microvascular endothelial cells (HRMECs) exposed to rapamycin under physiological $(5.5 \mathrm{mM})$ and high- glucose $(25 \mathrm{mM})$ conditions. Flow cytometry analyses showed significant increases in intracellular ROS levels in HRMECs under a high-glucose condition, and these increases were significantly suppressed by rapamycin $(P<0.0083$, the Bonferroni correction).

In STZ-injected hyperglycemic rats which VEGF increases in the retina, this mTOR is amplified ${ }^{20}$. In the present study, rapamycin suppressed the increase of VEGF in the diabetic retina (Figs. 1, 2, 3) and prevented the hyperglycemia-induced ROS level in both cultured Müller cells (Fig. 5) and primary HRMECs (Fig. 6). This indicated that rapamycin seemed to decrease ROS levels by inhibiting the mTOR pathway in retinal Müller cells; however, we did not assess its effects on non-Müller rat retinal cells. Our results are consistent with co-localization being at least in part within the Müller cells, and it is not excluded from the present study that some non-Müller cells may also co-express VEGF and GFAP. In the retina, Müller cells and vascular endothelial cells might express mTOR.

ROS production, oxidative stress, and cellular death can be altered by several signaling pathways triggered by the hyperglycemia that causes diabetic retinopathy ${ }^{37}$. Our data indicated that VEGF-induced increase of the ROS levels in Müller cells under high-glucose conditions and this increase was suppressed by rapamycin. We also analyzed the intracellular ROS levels in primary HRMECs which have the potential to cause retinal vascularization observed in human diabetic retinopathy. Our findings indicate that rapamycin might be an effective alternative drug for the treatment of diabetic macular edema.

We also showed that GFAP, a glial marker, increased in the diabetic retina. Our findings showed that rapamycin can reduce the increase in GFAP expression in the diabetic retina, suggesting that mTOR inhibition may inhibit glial activation and/or proliferation. In non-diabetic rats, the effect of rapamycin on GFAP levels was observed as well. Rapamycin might inhibit the activity of retinal glia even also under the physiological condition. In diabetic retinopathy, neurodegeneration starts even at an early stage of the disorder where reactive gliosis plays a crucial role ${ }^{17,38}$. It is known that there are two types of mTOR, mTORC1 (mainly retinal ganglion cells) and mTORC2 (mainly glial cells) in the retina ${ }^{39-41}$. The effects of rapamycin in this study seem to be caused mainly by mTORC2 inhibition in the diabetic rat retina.

A limitation of this study is that although the chief focus was on oft-studied rat models of diabetes, it will be of importance for future analyses to extend analyses to the retinas of animals and humans exhibiting anatomi$\mathrm{cal}$ and functional manifestations of diabetic retinopathy. Another possible limitation is that although mTOR is reported to increase in the retinas of rats with experimental diabetes ${ }^{20}$, this remains to be confirmed in retinas of rats made hyperglycemic by STZ for 8 weeks. Thirdly, although FACS was used to quantify ROS in cultured retinal Müller cells and HRMECs, it remains to be demonstrated in future studies that rapamycin suppresses excessive ROS production in vivo within the diabetic retina.

In conclusion, our findings from in vivo and in vitro experiments showed that the mTOR inhibitor, rapamycin, blocks the up-regulation of VEGF and GFAP in the diabetic rat retina and also inhibits the hyperglycemiainduced increase in ROS in cultured Müller cells and HERMCs. These findings suggest that rapamycin may be a potential candidate drug for ameliorating retinal complications in diabetes.

Received: 24 June 2020; Accepted: 7 January 2021

Published online: 21 January 2021

\section{References}

1. Targher, G., Bertolini, L., Zoppini, G., Zenari, L. \& Falezza, G. Increased plasma markers of inflammation and endothelial dysfunction and their association with microvascular complications in Type 1 diabetic patients without clinically manifest macroangiopathy. Diabet. Med. 22, 999-1004. https://doi.org/10.1111/j.1464-5491.2005.01562.x (2005).

2. Kohner, E. M. Microvascular disease: What does the UKPDS tell us about diabetic retinopathy?. Diabet. Med. 25(Suppl 2), 20-24. https://doi.org/10.1111/j.1464-5491.2008.02505.x (2008).

3. Rask-Madsen, C. \& King, G. L. Vascular complications of diabetes: Mechanisms of injury and protective factors. Cell Metab. 17, 20-33. https://doi.org/10.1016/j.cmet.2012.11.012 (2013).

4. Whitmire, W., Al-Gayyar, M. M., Abdelsaid, M., Yousufzai, B. K. \& El-Remessy, A. B. Alteration of growth factors and neuronal death in diabetic retinopathy: What we have learned so far. Mol. Vis. 17, 300-308 (2011).

5. Busch, S. et al. Systemic treatment with erythropoietin protects the neurovascular unit in a rat model of retinal neurodegeneration. PLoS ONE 9, e102013. https://doi.org/10.1371/journal.pone.0102013 (2014). 
6. Bandello, F. et al. Retinal layer location of increased retinal thickness in eyes with subclinical and clinical macular edema in diabetes type 2. Ophthalmic Res. 54, 112-117. https://doi.org/10.1159/000438792 (2015).

7. Zhou, X., Ai, S., Chen, Z. \& Li, C. Probucol promotes high glucose-induced proliferation and inhibits apoptosis by reducing reactive oxygen species generation in Müller cells. Int. Ophthalmol. 39, 2833-2842. https://doi.org/10.1007/s10792-019-01130-8 (2019).

8. Wu, M. Y., Yiang, G. T., Lai, T. T. \& Li, C. J. The oxidative stress and mitochondrial dysfunction during the pathogenesis of diabetic retinopathy. Oxid. Med. Cell. Longev. 2018, 3420187. https://doi.org/10.1155/2018/3420187 (2018).

9. Toda, N. \& Nakanishi-Toda, M. Nitric oxide: ocular blood flow, glaucoma, and diabetic retinopathy. Prog. Retin Eye Res. 26, 205-238. https://doi.org/10.1016/j.preteyeres.2007.01.004 (2007).

10. Cheng, Z. et al. Betulinic acid derivatives can protect human Müller cells from glutamate-induced oxidative stress. Exp. Cell Res. 383, 111509. https://doi.org/10.1016/j.yexcr.2019.111509 (2019).

11. Wang, J., Xu, X., Elliott, M. H., Zhu, M. \& Le, Y. Z. Müller cell-derived VEGF is essential for diabetes-induced retinal inflammation and vascular leakage. Diabetes 59, 2297-2305. https://doi.org/10.2337/db09-1420 (2010).

12. Pierce, E. A., Avery, R. L., Foley, E. D., Aiello, L. P. \& Smith, L. E. Vascular endothelial growth factor/vascular permeability factor expression in a mouse model of retinal neovascularization. Proc. Natl. Acad. Sci.U S A 92, 905-909. https://doi.org/10.1073/ pnas.92.3.905 (1995)

13. Lim, Y. C. et al. Prevention of VEGF-mediated microvascular permeability by C-peptide in diabetic mice. Cardiovasc. Res. 101, 155-164. https://doi.org/10.1093/cvr/cvt238 (2014).

14. Ma, M. et al. High glucose-induced TRPC6 channel activation decreases glutamate uptake in rat retinal müller cells. Front. Pharmacol. 10, 1668. https://doi.org/10.3389/fphar.2019.01668 (2019).

15. Chidlow, G., Wood, J. P. M. \& Casson, R. J. Investigations into hypoxia and oxidative stress at the optic nerve head in a rat model of glaucoma. Front. Neurosci. 11, 478. https://doi.org/10.3389/fnins.2017.00478 (2017).

16. Yabana, T. et al. The relationship between glutathione levels in leukocytes and ocular clinical parameters in glaucoma. PLoS ONE 14, e0227078. https://doi.org/10.1371/journal.pone.0227078 (2019).

17. Simó, R., Stitt, A. W. \& Gardner, T. W. Neurodegeneration in diabetic retinopathy: Does it really matter?. Diabetologia 61, 19021912. https://doi.org/10.1007/s00125-018-4692-1 (2018).

18. Antonetti, D. A., Klein, R. \& Gardner, T. W. Diabetic retinopathy. N. Engl. J. Med. 366, 1227-1239. https://doi.org/10.1056/NEJMr a1005073 (2012).

19. Araki, E. \& Nishikawa, T. Oxidative stress: A cause and therapeutic target of diabetic complications. J, Diabet. Investig. 1, 90-96. https://doi.org/10.1111/j.2040-1124.2010.00013.x (2010).

20. Wei, J., Jiang, H., Gao, H. \& Wang, G. Blocking mammalian target of rapamycin (mTOR) attenuates HIF-1alpha pathways engaged-vascular endothelial growth factor (VEGF) in diabetic retinopathy. Cell Physiol. Biochem. 40, 1570-1577. https://doi. org/10.1159/000453207 (2016).

21. Benjamin, D., Colombi, M., Moroni, C. \& Hall, M. N. Rapamycin passes the torch: A new generation of mTOR inhibitors. Nat. Rev. Drug Discov. 10, 868-880. https://doi.org/10.1038/nrd3531 (2011).

22. Ozdemir, G., Kilinc, M., Ergun, Y. \& Sahin, E. Rapamycin inhibits oxidative and angiogenic mediators in diabetic retinopathy. Can. J. Ophthalmol. 49, 443-449. https://doi.org/10.1016/j.jcjo.2014.07.003 (2014).

23. Kilkenny, C., Browne, W. J., Cuthill, I. C., Emerson, M. \& Altman, D. G. Improving bioscience research reporting: The ARRIVE guidelines for reporting animal research. PLoS Biol. 8, e1000412. https://doi.org/10.1371/journal.pbio.1000412 (2010).

24. Guo, N. et al. The protective effect and mechanism of rapamycin in the rat model of IgA nephropathy. Ren. Fail. 41, 334-339. https ://doi.org/10.1080/0886022x.2019.1577257 (2019).

25. Erlich, S., Alexandrovich, A., Shohami, E. \& Pinkas-Kramarski, R. Rapamycin is a neuroprotective treatment for traumatic brain injury. Neurobiol. Dis. 26, 86-93. https://doi.org/10.1016/j.nbd.2006.12.003 (2007).

26. Oku, H. et al. Tau is involved in death of retinal ganglion cells of rats from optic nerve crush. Invest. Ophthalmol. Vis. Sci. 60, 2380-2387. https://doi.org/10.1167/iovs.19-26683 (2019).

27. Kida, T. et al. Implication of VEGF and aquaporin 4 mediating Muller cell swelling to diabetic retinal edema. Graefe Arch. Clin. Exp. Ophthalmol. 255, 1149-1157. https://doi.org/10.1007/s00417-017-3631-z (2017).

28. Suzuki, H. et al. Changes in expression of aquaporin-4 and aquaporin-9 in optic nerve after crushing in rats. PLoS ONE 9, e114694. https://doi.org/10.1371/journal.pone.0114694 (2014).

29. Yanai, N. \& Obinata, M. Apoptosis is induced at nonpermissive temperature by a transient increase in p53 in cell lines immortalized with temperature-sensitive SV40 large T-antigen gene. Exp. Cell Res. 211, 296-300. https://doi.org/10.1006/excr.1994.1090 (1994).

30. Abukawa, H. et al. Modulation of retinal capillary endothelial cells by Müller glial cell-derived factors. Mol. Vis. 15, 451-457 (2009).

31. Bindokas, V. P., Jordan, J., Lee, C. C. \& Miller, R. J. Superoxide production in rat hippocampal neurons: Selective imaging with hydroethidine. J. Neurosci. 16, 1324-1336 (1996).

32. Zhao, H. et al. Detection and characterization of the product of hydroethidine and intracellular superoxide by HPLC and limitations of fluorescence. Proc. Natl. Acad. Sci. U S A 102, 5727-5732. https://doi.org/10.1073/pnas.0501719102 (2005).

33. Arden, G. B. \& Sivaprasad, S. Hypoxia and oxidative stress in the causation of diabetic retinopathy. Curr. Diabetes Rev. 7, 291-304 (2011).

34. Kida, T. et al. NADPH oxidase-mediated ROS production determines insulin's action on the retinal microvasculature. Invest. Ophthalmol. Vis. Sci. 56, 6754-6761. https://doi.org/10.1167/iovs.15-17534 (2015).

35. Islam, M. T. Oxidative stress and mitochondrial dysfunction-linked neurodegenerative disorders. Neurol. Res. 39, 73-82. https:// doi.org/10.1080/01616412.2016.1251711 (2017).

36. Morita, M. et al. mTOR coordinates protein synthesis, mitochondrial activity and proliferation. Cell Cycle 14, 473-480. https:// doi.org/10.4161/15384101.2014.991572 (2015).

37. Volpe, C. M. O., Villar-Delfino, P. H., Dos Anjos, P. M. F. \& Nogueira-Machado, J. A. Cellular death, reactive oxygen species (ROS) and diabetic complications. Cell Death Dis. 9, 119. https://doi.org/10.1038/s41419-017-0135-z (2018).

38. Tzekov, R. \& Arden, G. B. The electroretinogram in diabetic retinopathy. Surv. Ophthalmol. 44, 53-60. https://doi.org/10.1016/ s0039-6257(99)00063-6 (1999).

39. Fort, P. E. et al. mTORC1-independent reduction of retinal protein synthesis in type 1 diabetes. Diabetes 63, 3077-3090. https:// doi.org/10.2337/db14-0235 (2014).

40. Nakahara, T., Morita, A., Yagasaki, R., Mori, A. \& Sakamoto, K. Mammalian target of rapamycin (mTOR) as a potential therapeutic target in pathological ocular angiogenesis. Biol. Pharm. Bull. 40, 2045-2049. https://doi.org/10.1248/bpb.b17-00475 (2017).

41. Losiewicz, M. K. et al. mTORC1 and mTORC2 expression in inner retinal neurons and glial cells. Exp. Eye Res. 197, 108131. https ://doi.org/10.1016/j.exer.2020.108131 (2020).

\section{Acknowledgements}

The authors thank Prof. Donald Puro for helpful comments on this paper. 


\section{Author contributions}

T.K., S.O., and T.H. performed the experiments. T.K. wrote the main manuscript text and T.K. and T.H. prepared all figures. H.O. and T.I. oversaw the project and assisted with the writing of the manuscript.

\section{Funding}

Financial support included Grant-in-Aid for Scientific Research (C) (Researcher No. 90610105) from the Japan Society for the Promotion of Science (Tokyo, Japan), and funding from the Osaka Eye Bank Association Fund (Osaka, Japan). The sponsors had no role in the design or conduct of this research.

\section{Competing interests}

The authors declare no competing interests.

\section{Additional information}

Supplementary Information The online version contains supplementary material available at https://doi. org/10.1038/s41598-021-81482-3.

Correspondence and requests for materials should be addressed to T.K.

Reprints and permissions information is available at www.nature.com/reprints.

Publisher's note Springer Nature remains neutral with regard to jurisdictional claims in published maps and institutional affiliations.

(c) (i) Open Access This article is licensed under a Creative Commons Attribution 4.0 International License, which permits use, sharing, adaptation, distribution and reproduction in any medium or format, as long as you give appropriate credit to the original author(s) and the source, provide a link to the Creative Commons licence, and indicate if changes were made. The images or other third party material in this article are included in the article's Creative Commons licence, unless indicated otherwise in a credit line to the material. If material is not included in the article's Creative Commons licence and your intended use is not permitted by statutory regulation or exceeds the permitted use, you will need to obtain permission directly from the copyright holder. To view a copy of this licence, visit http://creativecommons.org/licenses/by/4.0/.

(C) The Author(s) 2021 